\title{
The chemical composition of galactic cosmic rays during solar minimum of solar cycle 20/21 - Helios E6 results
}

Johannes Marquardt;, Bernd Heber, Malte Hörlöck, Patrick Kühl, Robert F. Wimmer-Schweingruber

CAU-Kiel, Germany

E-mail: marquardtephysik.uni-kiel.de

In October 2011, ESA announced the selection of Solar Orbiter as one of the Cosmic Vision M missions. It's launch is foreseen in 2018. Therefore it is worthwhile to revisit the energetic particle measurements together with the solar wind and magnetic field data from Helios in light of better theoretical understanding and advanced analysis and modelling techniques developed during the past 20 years. In this contribution we will present a GEANT 4 simulation of the response function of the Helios E6 experiment, that measured electrons in the energy range from a few $100 \mathrm{keV}$ to above $10 \mathrm{MeV}$, ions from $1 \mathrm{MeV} /$ nucleon to above $50 \mathrm{MeV} /$ nucleon, and its application to the data analysis with respect to the chemical composition of the Galactic Cosmic Rays.

The 34th International Cosmic Ray Conference

30 July- 6 August, 2015

The Hague, The Netherlands

\footnotetext{
*Speaker.
} 
Table 1: Relative Elemental Abundances at $160 \mathrm{MeV} / \mathrm{Nucleon}$ from ACE/CRIS [6, 9]

\begin{tabular}{cccc} 
Element & $\begin{array}{c}\text { Solar Minimum } \\
1997 / 1998[6]\end{array}$ & $\begin{array}{c}\text { Solar Minimum } \\
\text { 2009-2010 [9] } \\
\text { at 160 MeV/nucleon }\end{array}$ & $\begin{array}{c}\text { Solar Maximum } \\
1998-2001[6]\end{array}$ \\
\hline $\mathrm{B}$ & $1803.8 \pm 10.4$ & $1725.7 \pm 19.4$ & $1986.4 \pm 11.3$ \\
$\mathrm{C}$ & $7337.0 \pm 18.4$ & $7235.4 \pm 45$ & $6780.2 \pm 18.4$ \\
$\mathrm{~N}$ & $1713.7 \pm 8.4$ & $1678.9 \pm 12.3$ & $1836.1 \pm 9$ \\
$\mathrm{O}$ & $7082.6 \pm 16$ & $7137.0 \pm 42.7$ & $6520.6 \pm 15.6$ \\
$\mathrm{Ne}$ & $998.7 \pm 5.6$ & $998.9 \pm 8.4$ & $1050 \pm 5.8$ \\
$\mathrm{Mg}$ & $1368 \pm 6.1$ & $1375.3 \pm 10.3$ & $1367.3 \pm 6$ \\
$\mathrm{Si}$ & $1000 \pm 5$ & $1000 \pm 7.8$ & $1000 \pm 4.8$ \\
\hline
\end{tabular}

\section{Introduction}

The elemental composition of the cosmic rays in the heliosphere has been measured a number of times during different phases in the solar cycle $[4,6]$ and different radial distances from the Sun beyond the Earth orbit $[5,3]$.

One of these measurements was carried out by the Experiment 6 (E6) on Helios. Helios 1 and Helios 2 were launched on December 10, 1974 and January 15, 1976, respectively. The two almost identical space probes were sent into ecliptic orbits around the Sun. The orbital period around the Sun was 190 days for Helios 1 and 185 days for Helios 2. Their perihelia were 0.3095 AU and 0.290 AU, respectively.

The E6 particle telescope relies on the $\frac{d E}{d x}-E$-method e.g.[10]. In order to interpret the measured data a detailed understanding of the instrument is needed and can be obtained by modelling the physical processes inside the detector taking into account the instrument geometry as well as environment e.g. [7].

The instrument consists of a stack of 5 semiconductor, a sapphire and a plastic scintillation detector (see Fig. 1), which measures the number of particles hitting each detector and their energy losses. A rough particle identification (species, energy and incoming direction) can be obtained by analyzing the count rate data that are obtained in (anti)-coincidence of a set of active detectors [8]. For a statistical ensemble not only the (anti-)coincidence conditions but also the energy loss in the last three detectors are known. The channel that is analyzed here in detail counts particles in the energy range from $\sim 13 \mathrm{MeV}$ /nucleon to $\sim 27 \mathrm{MeV}$ /nucleon protons and helium.

In order to determine the chemical composition of cosmic rays, the detailed instrument response for different particle species is calculated utilizing a newly developed model of the E6 instrument. The calculations are based on the program library Geant 4 that is a toolkit for the simulation of the passage of particles through matter [1]. The purpose of this contribution is to show that the Helios E6-Experiment is capable of determining the chemical composition of particles that are stopped in the detector sensor up to silicon. 


\section{Instrumentation}

The Kiel experiment E6 for studying energetic cosmic rays was built as an universal detector. It was supposed to measure most effects of the cosmic radiation which occur in interplanetary space. The energy range extends from $1.3 \mathrm{MeV} / \mathrm{n}$ to $>1000 \mathrm{MeV} / \mathrm{n}$ for nuclei and from approximately 0.3 to $8 \mathrm{MeV}$ for electrons. The on-board data processing system evaluates the measured pulses created during the particle transition.

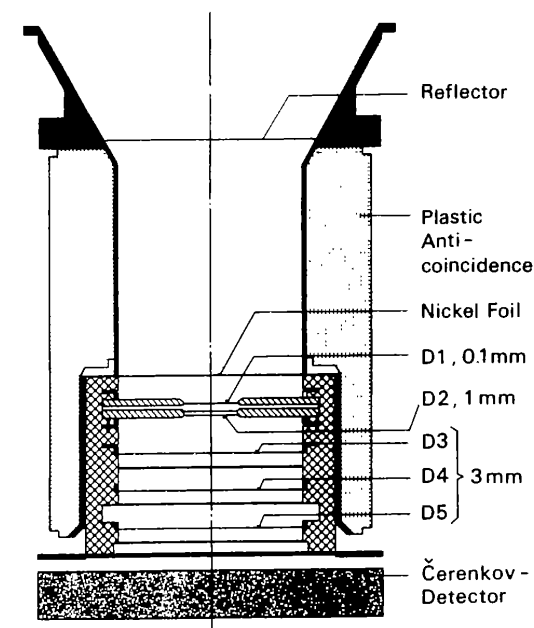

Figure 1: Schematic of the Helios E6

The detector system consists of five semiconductor-detectors of increasing thickness that are shown in Fig. 1. The figure also indicates the thicknesses of the detectors. While detectors D1(A) and D2(B) are silicon-surface-barrier-detectors with a thickness of 100 and $1000 \mu \mathrm{m}$, the other three are lithium drifted detectors with a thickness of $3000 \mu \mathrm{m}$. The first two (D1 and D2) are used for the determination of the lowest energy channels, for the definition of the geometry factor for stopping particles (energy ranges below $51 \mathrm{MeV} / \mathrm{N}$ ) as well as for the discrimination between electrons and nuclei. The first detector does not respond to relativistic electrons (above 300 $\mathrm{keV}$ ), the discrimination between electrons and nuclei is that the first detector is not triggered for electrons. To avoid false identifications by these discrimination conditions, the first and second detectors have been placed on top of each other, as close as possible. Charged particles, which penetrate the fifth detector and the aluminum absorber beneath, are detected in the Sapphire-Cerenkovdetector. The Cerenkov-threshold for this material $(\mathrm{n}=1.8)$ is $E_{s}=210 \mathrm{MeV} /$ nucleon. Because the Sapphire-Cerenkov-detector also delivers scintillation light, particles in the energy range above 51 $\mathrm{MeV} /$ nucleon are counted in an integral channel.

\section{Results}

Energy spectra for galactic and if present, anomalous, protons, helium, carbon, nitrogen, and oxygen in the energy range from a few $\mathrm{MeV} /$ nucleon to several $100 \mathrm{MeV} /$ nucleon were determined by Christian 1989, [2]. Fig. 2 displays 12 hour averaged count rates of $\sim 13$ to $\sim 27 \mathrm{MeV} /$ nucleon protons (black curve) and ions (red curve) from Helios 1 and Helios 2 from launch to September 


\begin{tabular}{ccccc} 
Element & Position & $\sigma$ & Total number & relative abundance $(\mathrm{Si})$ \\
\hline helium & 3.27 & 0.03 & 11849 & 95500 \\
boron & 4.52 & 0.03 & 71 & 570 \\
carbon & 4.73 & 0.03 & 348 & 2790 \\
nitrogen & 4.94 & 0.03 & 102 & 820 \\
oxygen & 5.11 & 0.03 & 460 & 3700 \\
neon & 5.42 & 0.03 & 89 & 720 \\
magnesium & 5.66 & 0.03 & 119 & 960 \\
silicon & 5.86 & 0.03 & 124 & 1000 \\
\hline
\end{tabular}

Table 2: Results of the fit using a Gaussion to the data in Fig.3. The position and $\sigma$ give the position of the maximum and the width of the Gaussian in $\log \left(E_{B} \cdot E_{t o t} / \mathrm{MeV}^{2}\right)$. The total number is calculated by integration of the Gaussian function. The relative abundance is given with respect to silicon.

1977, respectively. For our analysis only data with a proton count rate below 0.002 counts/second was used.

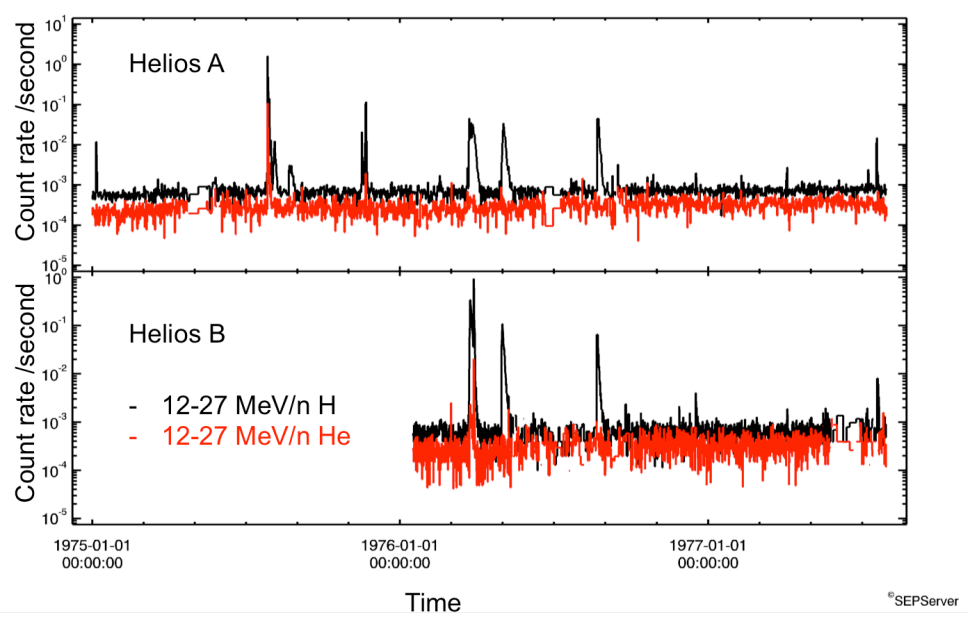

Figure 2: The upper and lower panel display the 12 hour averaged count rates of $\sim 13$ to $\sim 27 \mathrm{MeV} /$ nucleon protons (black curve) and ions (red curve) from Helios 1 and Helios 2 from launch to September 1977, respectively. For details see text.

The lower panel of Fig. 3 shows the simulated $\Delta E \cdot E$ distribution for helium and above. From that figure we find peaks that correspond to boron, carbon, nitrogen, oxygen, and neon. In order to determine the abundances, each peak was fitted with a corresponding Gaussian function. The corresponding lower panel shows the fit residuals which demonstrate the goodness of the fit. We find a typical width $\sigma$ of 0.03 that allows us to separate between $\mathrm{C}, \mathrm{N}, \mathrm{O}$, and $\mathrm{Ne}$ by more than $3 \sigma$. The same was done with the measured data, leading to the results summarized in Tab. 2. In order to estimate the elemental separation of the instrument the figure displays the Gaussian fits for B, C, $\mathrm{N}, \mathrm{O}, \mathrm{Ne}, \mathrm{Mg}$, and $\mathrm{Si}$. From this the background distribution can be analyzed further and the edge effect and possible further nuclei like ${ }^{3} \mathrm{He}$ can be investigated in more detail. The total number of counts varies between 70 for boron and 12000 for helium. Since abundances are given relative to 
silicon, the statistical accuracy cannot be better than $10 \%$ for helium and increases to more than $15 \%$ for boron. However, the relative abundances differ significantly from the ones given in Tab. 1 . This can be explained mainly by the fact that in contrast to the work by [6] the values are not normalized to a reference energy. While the channel analyzed here covers an energy range from 13 to $27 \mathrm{MeV} /$ nucleon for helium, it measures heavier elements at much higher energies. Thus an extended analysis is needed that includes other energy channels from the Helios instrument in order to define a single energy range that is covered by all species. 

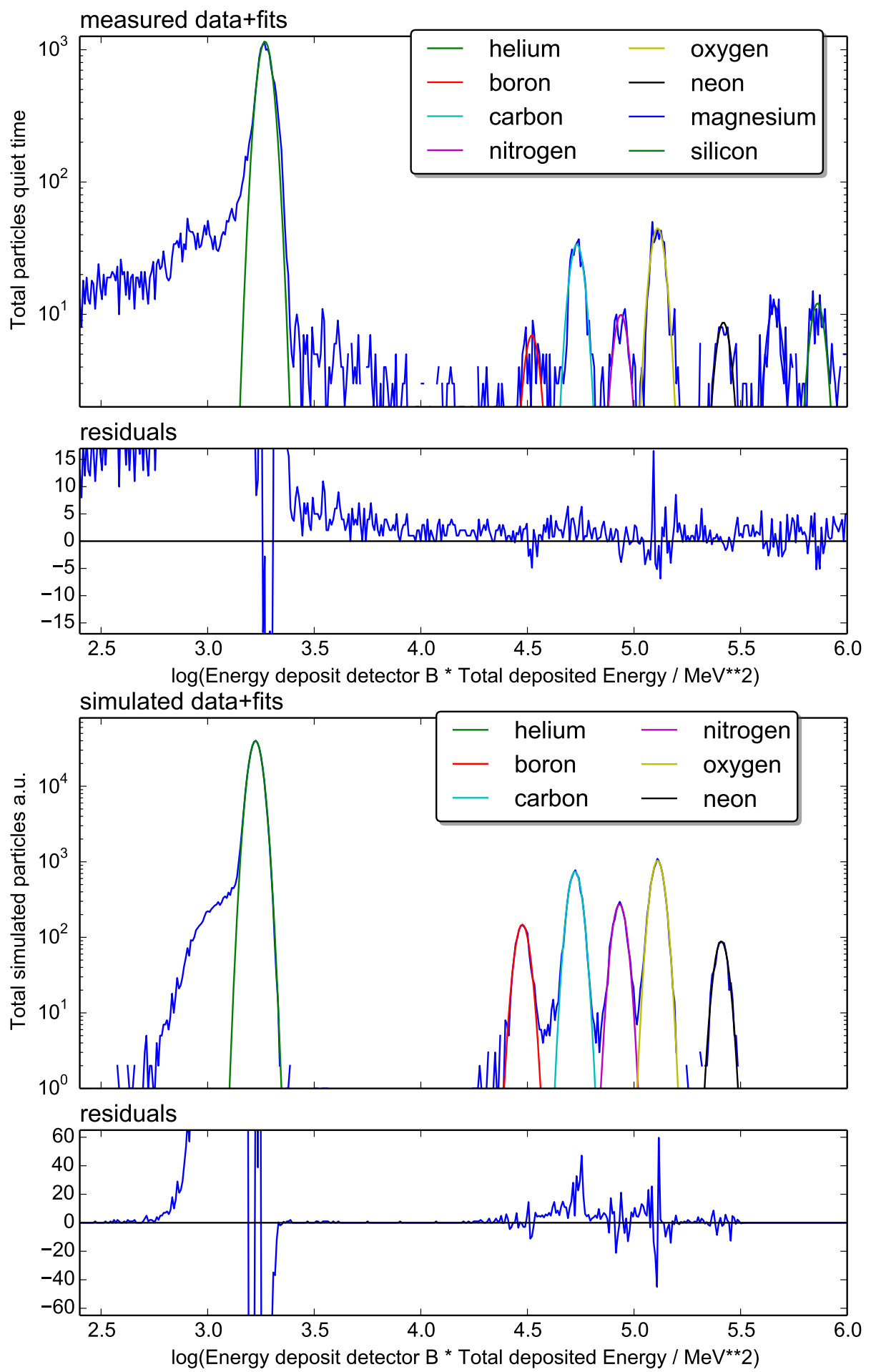

Figure 3: The blue curves show the left projection (1-dimensional representation) of the different measured elements and simulated distribution. The other colored curves are the result of fits by a Gaussian to the data. The bottom panels display the residuals of the fit, indicating the goodness of the fit. Note that the y-axis are on logarithmic and linear scale on the top and bottom, respectively. 


\section{References}

[1] S. etal. Agostinelli, Geant 4, a simulation toolkit, Nuclear Instruments and Methods in Physics Research Section A: Accelerators, Spectrometers, Detectors and Associated Equipment 506 (2003), no. 3, 250-303.

[2] E. R. Christian, Evidence for anomalous cosmic ray hydrogen, Ph.D. thesis, California Institute of Technology, Pasadena., 1989.

[3] M. A. Duvernois and M. R. Thayer, The Elemental Composition of the Galactic Cosmic-Ray Source: ULYSSES High-Energy Telescope Results, 465 (1996), 982.

[4] J. J. Engelmann, P. Ferrando, A. Soutoul, P. Goret, and E. Juliusson, Charge composition and energy spectra of cosmic-ray nuclei for elements from Be to NI - Results from HEAO-3-C2, 233 (1990), 96-111.

[5] P. Ferrando, N. Lal, F. B. McDonald, and W. R. Webber, Studies of low-energy Galactic cosmic-ray composition at 22 AU. I - Secondary/primary ratios, 247 (1991), 163-172.

[6] J. S. George, K. A. Lave, M. E. Wiedenbeck, W. R. Binns, A. C. Cummings, A. J. Davis, G. A. de Nolfo, P. L. Hink, M. H. Israel, R. A. Leske, R. A. Mewaldt, L. M. Scott, E. C. Stone, T. T. von Rosenvinge, and N. E. Yanasak, Elemental Composition and Energy Spectra of Galactic Cosmic Rays During Solar Cycle 23, 698 (2009), 1666-1681.

[7] B Heber, A Kopp, H Fichtner, and S E S Ferreira, On the determination of energy spectra of MeV electrons by the Ulysses COSPIN/KET, Advances in Space Research 35 (2005), no. 4, 605-610.

[8] H. Kunow, G. Wibberenz, G. Green, R. Müller-Mellin, and M.-B. Kallenrode, Energetic Particles in the Inner Solar System, Physics of the Inner Heliosphere II. Series: Physics and Chemistry in Space 21 (1991), 243-342.

[9] K. A. Lave, M. E. Wiedenbeck, W. R. Binns, E. R. Christian, A. C. Cummings, A. J. Davis, G. A. de Nolfo, M. H. Israel, R. A. Leske, R. A. Mewaldt, E. C. Stone, and T. T. von Rosenvinge, Galactic Cosmic-Ray Energy Spectra and Composition during the 2009-2010 Solar Minimum Period, 770 (2013), 117.

[10] F. B. McDonald and G. H. Ludwig, Measurement of Low-Energy Primary Cosmic-Ray Protons on IMP-1 Satellite, Physical Review Letters 13 (1964), 783-785. 